\title{
DPD Linearization Complexity Reduction of Remote Radio Heads in C-RAN with Radio over Fiber Fronthaul
}

\author{
Pedro Luis Carro, Carlos Mateo, Paloma García-Dúcar, Jesús de Mingo, Antonio Valdovinos \\ Department of Electronic Engineering and Communications \\ Aragon Institute of Engineering Research (I3A) \\ University of Zaragoza, Zaragoza, 50.018, Spain \\ Email: plcarro@unizar.es,cmperez@unizar.es,paloma@unizar.es, mingo@unizar.es,toni@unizar.es
}

\begin{abstract}
Radio-over-Fiber (RoF) is a suitable technology for efficiently developing the cloud-RAN concept in Fifth Generation (5G) deployments based on Long Term Evolution Advanced LTE-A. Distortion coming out from the radiofrequency (RF) power amplifier should be taken into account in order to achieve the desired performance at the base station. In this paper, a complexity reduction technique based on a variable degree polynomial predistorter is proposed. The obtained results show that it is possible to achieve a reduction of operations with a penalty of 1.6 dB in ACLR and negligible impact on the EVM.

Keywords - Long-Term Evolution (LTE), Digital
\end{abstract} Predistortion, Radio-over- Fiber (RoF), C-RAN

\section{INTRODUCTION}

The growth experienced in recent years by data traffic resulting from the expansion of mobile services and the prospects that this growth will significantly increase in the near future require new solutions that combine a more efficient use of the spectrum and an efficient use of the latest technological advances in signal processing. This is the basis for future $5 \mathrm{G}$ communications systems, where the concept of cloud-RAN will be applied [1]. In cloud RAN architectures, the functionalities of the base stations (BS) are split in two levels: a centralized baseband unit (BBU), of great capacity of computation and a set of distributed Remote Radio Head (RRH) units. Current OFDM-based mobile communications systems like LTE-A, are very sensitive to the nonlinear distortions introduced by the analogue parts, especially at the transmitter side. The power amplifier nonlinear behavior will now add a new source of distortion due to the electric to optical and optical to electric converters and the fiber dispersion [2]. Among all PA linearization methods, digital predistortion (DPD) [3] becomes one of the most cost-effective due to its high precision and relative simplicity.

Models used in DPD coefficients extraction are usually based on mathematical methods which arise in the description of nonlinear systems. Most of them were developed using the Volterra series approach. This method provides a very powerful way to model PAs and extract their nonlinearities and memory effects. Unfortunately, the classical Volterra model is too complex and it is usually difficult to apply it in a real system. Hence, several simplified models have been developed to characterize PAs with enough accuracy under certain conditions. Polynomial models provide a significant complexity reduction while keeping a reasonable accuracy.

The paper is outlined as follows. In Section II, we introduce the proposed variable variable degree DPD to decrease the computational complexity. The experimental setup and the main results are discussed in section III. Finally, some conclusions about this work are provided in Section IV.

\section{DigitAl PREDistortion APPLICATION}

\section{A. DPD model description}

The predistortion model proposed in this work is based on the classical memory-polynomial-model (MPM) defined by

$$
u(n)=\sum_{m=0}^{N-1} x(n-m) \sum_{k=0}^{M-1} w_{k m}|x(n-m)|^{k},
$$

where $x(n)$ is the input signal, $u(n)$ the output signal, $N$ represents the non-linear order, and $M$ the memory depth of the mathematical model. Fig.1 shows the block diagram corresponding to a simplified C-RAN architecture including a Radio over Fiber link in order to transmit from the BBU to the RRH and the Power Amplifier (PA). The RoF link will introduce some distortion, but the main source of nonlinearity is the PA. In addition, it shows the indirect-learning structure used for predistorter identification. Using this scheme, in a first training stage, DPD coefficients are calculated in the feedback path (DPD extract block) whose input is $z(n)$, calculated as

$$
z(n)=y(n) / G_{\text {norm }},
$$

where $y(n)$ is the PA output, and $G_{\text {norm }}$ is the normalization gain of the linearized PA. Once DPD coefficients are calculated, the actual predistorter will be a copy of the DPD extract block. There are different approaches for estimating the model coefficients when applying DPD. The Least Squares solution corresponds to the minimization of the deterministic equation

$$
J(\mathbf{w})=\sum_{n=1}^{N}|e(n)|^{2},
$$




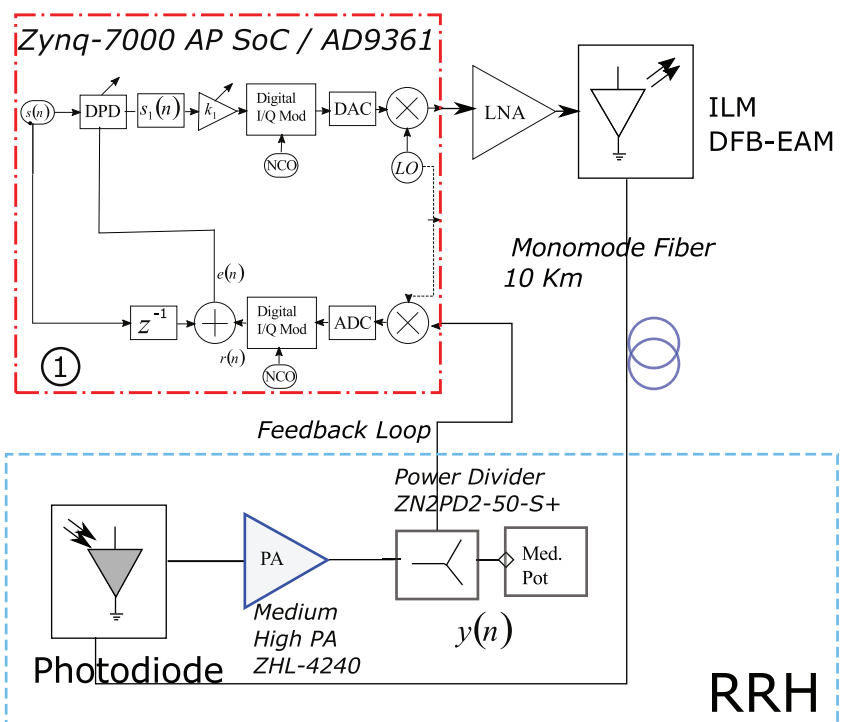

Fig. 1. Block diagram of the simplified C-RAN architecture.

where $e(n)=u(n)-\hat{u}(n)$. If we denote $\hat{\mathbf{u}}$ as the vector expression of $\hat{u}(n)$, the post-distorter output can be written as

$$
\hat{\mathbf{u}}=\mathbf{Z} \mathbf{w}
$$

considering $\mathbf{Z}$ the matrix expression of $z(n)$. The expression for the coefficient vector $\mathbf{w}$ using LS is well known [5]. Constructing properly the matrix data $\mathbf{Z}$ and the desired output vector $\mathbf{u}$, and following the proposed memoryless polynomial model, the LS solution for the DPD coefficients can be calculated as

$$
\mathbf{w}=\left(\mathbf{Z}^{H} \mathbf{Z}\right)^{-1} \mathbf{Z}^{H} \mathbf{u}
$$

where $(\cdot)^{H}$ represents the hermitian transpose operator, and the matrix product $\left(\mathbf{Z}^{H} \mathbf{Z}\right)^{-1} \mathbf{Z}^{H}$ is also known as the pseudoinverse of $\mathbf{Z}$.

The MPM previously assumes that the degree and the memory order are known or fixed in the design. The higher the order, the greater the complexity in terms of operations, which increase linearly with the order. Thus, every sample from the input signal is predistorted using the same number of coefficients. However, depending on the input envelop value, a lower degree MPM could be enough to compensate the nonlinearity, without an excessive loss in DPD performance.

Without loss of generality, we will focus on polynomial models without memory, but it is easy to generalize the method to include the delays. Based on eq.(1),

$$
u(n)=\sum_{k=0}^{f(|x|)} w_{k, f(|x|)} x(n)|x(n)|^{k}
$$

where $f(|x|)$ is a real function of integer value to be optimize which depends on the input envelope.

In order to compute this function, two steps are required. Firstly, a cost function which takes into account not only

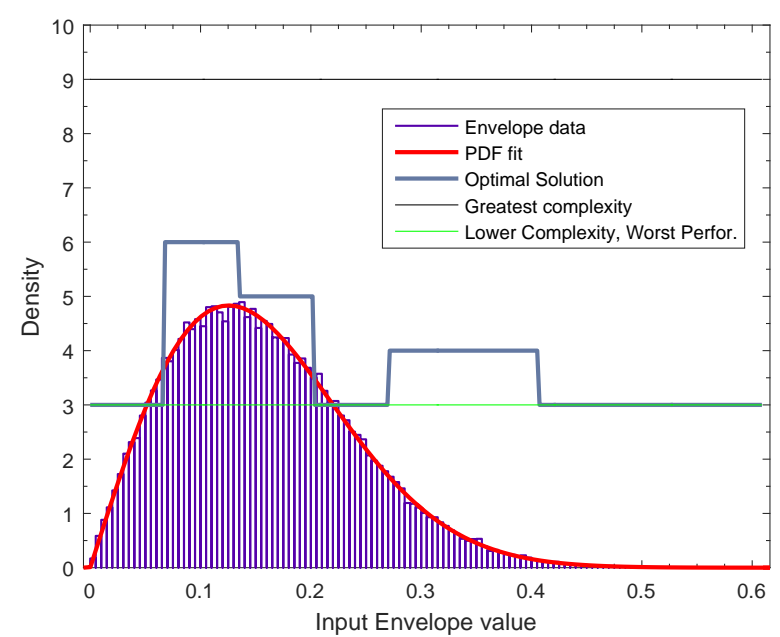

Fig. 2. Probability Desnsity Function and adaptive order proposed solution

performance but also complexity must be defined. Afterwards, a proper optimization algorithm should be carried out.

As far as the cost function is concerned, the polynomial degree should be constrained to a certain minimum and maximum value, $M_{\min }, M_{\max }$. Using the DPD coefficient computation, the DPDs of such degrees can be estimated and their performance can be evaluated with the aid of the least square error (MMSE), for example. Thus, using this collection of predistorters, the worst and best performance corresponding to , $M_{\min }, M_{\max }, e_{\min }$ and $e_{\max }$ are obtained.

The function $f(|x|)$ can be piecewise approximated by defining a (for example) uniform partition of the input envelope values $\left(0, \rho_{\max }=\max a b s(|x(n)|)\right)$,

$$
f(|x|)=\sum_{l=0 . . L-1} \alpha_{l} \prod\left(\frac{|x|-\left|x_{k}\right|}{\Delta}\right)
$$

being $\prod$ a pulse centered at $\left|x_{k}\right|$ and $\Delta$ width, being $\Delta$ the uniform step at the partition. Fig. 2 shows an example of such function together with the statistical distribution of a LTE signal, which fits to a Rayleigh distribution, as is a OFDM-like waveform. In addition, it shows the maximum complexity predistorter order which provides the best results in terms of performance and the lower complexity solution (order 3) which yields to the worst linearization. We propose a different order polynomial to be applied depending of the input sample, which can be computed using different methods. This leads to a reduction of multiplications to evaluate the polynomial. Although first the input must be classified into the right partition, this is a comparison which is equivalent to a sum operation, and therefore, the number of operations is decreased.

Eq. 6 can be applied to compute a variable degree polynomial model performance, $e$, and the performance efficiency can be defined as

$$
\eta_{l i n}=\frac{e_{\max }-e(f(|x|)}{e_{\max }-e_{\min }}
$$




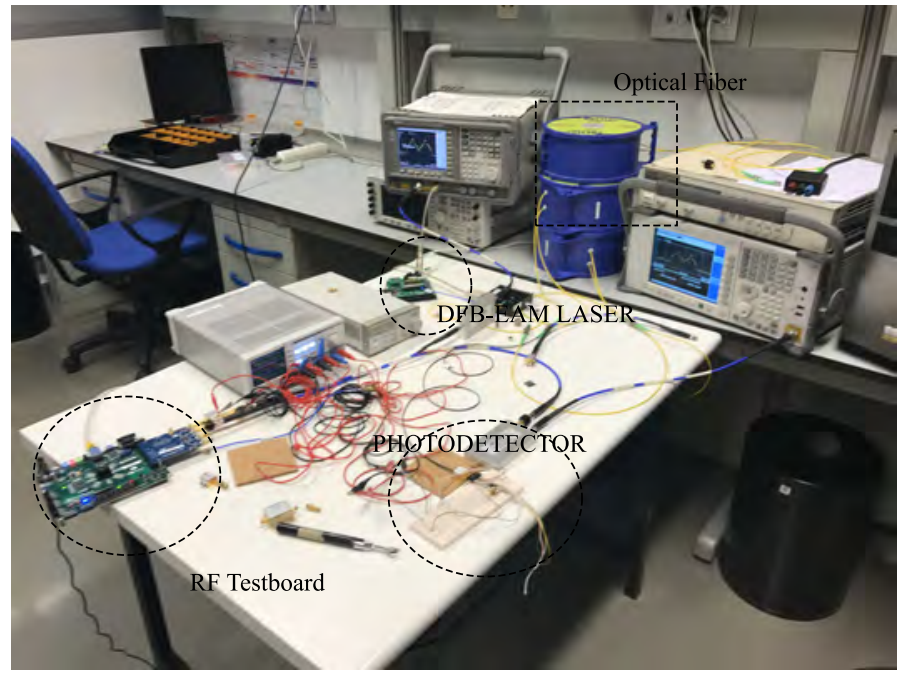

Fig. 3. Analog Optical Test-setup.

which ranges from 0 to 1 corresponding to $M_{\min }, M_{\max }$, respectively. In this work, the normalized mean square error (NMSE) is used to define the efficiency function. The normalized mean-square error is given by

$$
\mathrm{NMSE}=e(f|x|, L)=\frac{\sum_{i=1}^{N S}\left|y_{D P D}(i)-G_{n o r m} x(n)\right|^{2}}{\sum_{i=1}^{N S}\left|y_{D P D}(i)\right|^{2}}
$$

where $i$ specifies as sample and NS is the number of samples. This compares the actual predistorter output with the perfectly linearly amplified signal.

In addition, as the complexity is linear with the degree of the involved polynomial [4], we can define a complexity efficiency as:

$$
\eta_{\text {complex }}=\frac{\sum f(|x|)-M_{\max } L}{\left(M_{\min }-M_{\max }\right) L}
$$

This maps the highest constrained degree to 0 and the lowest constrained degree to $1 . L$ is the number of discretized values of the input envelope. The product of both efficiencies will be an utility function which takes into account the tradeoff between performance and complexity and it is useful to estimate $f|x|$.

The second step is to choose a integer optimization algorithm. As the number of variables is $\mathrm{L}$, the problem is complex. In this work we have selected a Integer Genetic (GA) algorithm, although there can be others to be applicable.

The integer GA will obtain the set of optimum degrees by maximizing

$$
\eta_{\text {TOTAL }}=\eta_{\text {lin }} \eta_{\text {complex }}
$$

\section{EXPERIMENTAL SETUP AND RESULTS}

\section{A. Experimental Setup}

The complete experimental test bench is shown in Fig. 1 through an equivalent block diagram including the real RF and optical elements. The optical part is showed in Fig. 3.

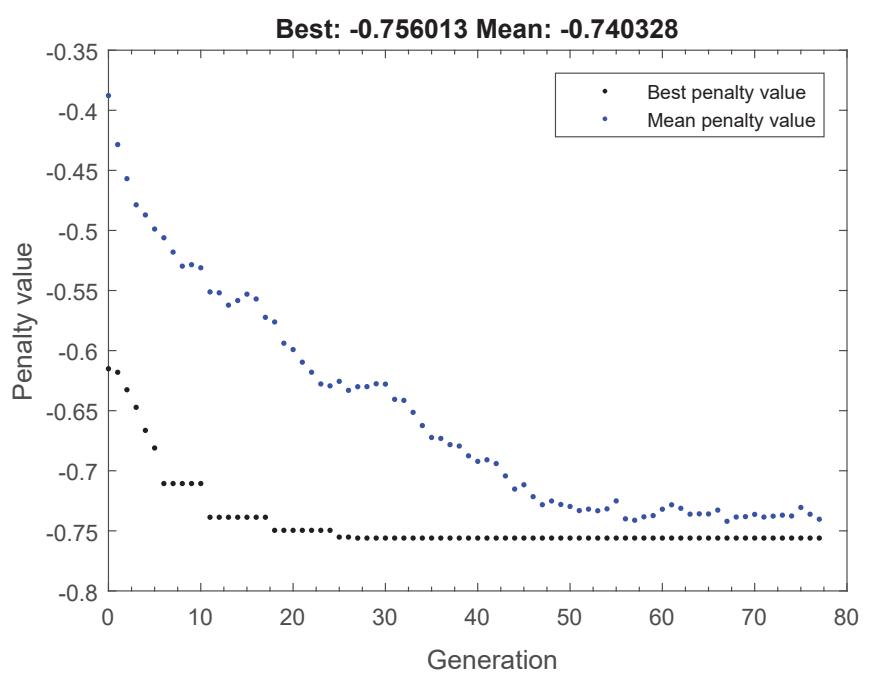

Fig. 4. Integer GA convergence with $L=9$.

The digital development platform used for the implementation of digital signal processes and the digital I/Q modulator and demodulator consists of a main board (ZedBoard featuring Zynq 7020 All-Programmable SoC) connected to a PC and to the Analog Devices ADFMCOMMS2-EBZ. The output signal of the DAC is preamplified and upconverted to a RF frequency of $1.8 \mathrm{GHz}$, within the Band 9 of the LTE standard for FDD duplex mode. The generated signal corresponds to a LTE downlink signal (OFDM modulation) with M-QAM modulated subcarriers and $5 \mathrm{MHz}$ bandwidth. The signal feeds a laser diode module. An electro-absorption modulator (EAM) Distributed Feed-Back (DFB) (Optilab DFB-EAM-1550-12 S/N7075), whose wavelength is $1550 \mathrm{~nm}$ is used in the experiments. The link between BBU and RRH is a single-mode fiber (SMF) with an attenuation of $0.25 \mathrm{~dB} / \mathrm{Km}$, a dispersion of $18 \mathrm{ps} /(\mathrm{nm}$ ?Km) and is $10 \mathrm{~km}$ length. The RRH side consists of a photodetector (PD) with a responsivity of 0.9 A/W (Nortel Networks PP-10G), and its output is amplified by a low noise amplifier (Mini Circuits ZX60-P33ULN+) and a PA (ZHL-4240) whose $1 \mathrm{~dB}$ compression point is $28 \mathrm{dBm}$. After the optic-electric conversion the signal is captured in the platform and analyzed in the computer.

\section{B. Results}

Fig. 4 shows the Integer GA convergence in the case of a partition with nine segments (i.e. nine variables to optimize) with $M_{\min }=3$ and $M_{\max }=9$ which is the worst case studied. The integer GA minimize $-\eta_{\text {total }}$ which is equivalent to maximize eq. 11. The algorithm finds the optimum in about 25 generations. Table I includes the performance in terms of efficiency, adjacent channel power ratio (ACPR) and Error Vector Magnitude (EVM). EVM provides a figure-of-merit for the system performance under test conditions. The received symbols $\left(y_{i}\right)$ are compared with the ideal symbols $\left(x_{i}\right)$ over a window of $N$ demodulated symbols. It is defined as 
Table 1. Optimum values for DPD and Performance Results

\begin{tabular}{|c|c|c|c|c|c|c|c|c|c|c|}
\hline $\mathrm{L}$ & $f(|x|), \alpha$ & $\eta_{\text {comp }}$ & $\eta_{l i n}$ & $\eta_{\text {total }}$ & input ACLR & ACLR no DPD & ACLR DPD & MMSE & EVM no DPD & EVM dpd \\
\hline 3 & 653 & 0.72 & 0.92 & 0.66 & 51.6 & 34.6 & 50.45 & -43.75 & $4.681 \%$ & $0.413 \%$ \\
\hline 4 & 6543 & 0.75 & 0.91 & 0.68 & 51.6 & 34.6 & 50.48 & -51.60 & $4.681 \%$ & $0.31 \%$ \\
\hline 5 & 66533 & 0.73 & 0.95 & 0.69 & 51.6 & 34.6 & 51.06 & -45.54 & $4.681 \%$ & $0.346 \%$ \\
\hline 6 & 663433 & 0.81 & 0.86 & 0.69 & 51.6 & 34.6 & 49.77 & -41.34 & $4.681 \%$ & $0.466 \%$ \\
\hline 7 & 3665333 & 0.81 & 0.88 & 0.71 & 51.6 & 34.6 & 50.08 & -42.23 & $4.681 \%$ & $0.345 \%$ \\
\hline 8 & 36454333 & 0.85 & 0.85 & 0.73 & 51.6 & 34.6 & 49.71 & -41.28 & $4.681 \%$ & $0.368 \%$ \\
\hline 9 & 365344333 & 0.87 & 0.86 & 0.75 & 51.6 & 34.6 & 50.02 & -41.51 & $4.681 \%$ & $0.342 \%$ \\
\hline
\end{tabular}
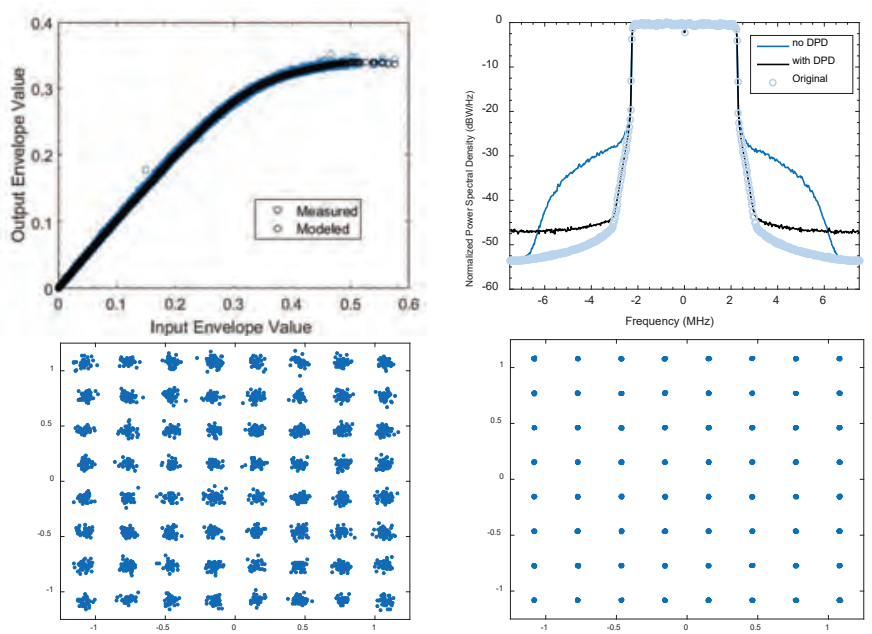

Fig. 5. System Behaviour and DPD Performance.

$$
\operatorname{EVM}(\%)=100 \cdot \sqrt{\frac{1}{N} \frac{\sum_{i=1}^{N}\left\|y_{i}-x_{i}\right\|^{2}}{\left\|y_{\max }\right\|^{2}}},
$$

where $y_{\max }$ is the outermost symbol in the constellation diagram and $N$ the number of complex data points used for computation. In addition, the Adjacent Channel Power Ratio (ACPR) quantifies spectral regrowth. It is used to measure the Adjacent Channel Interference (ACI), and is defined as

$$
\mathrm{ACPR}=\max _{m=1,2}\left[10 \log _{10} \frac{\int_{(\text {adj_band }) m} Y(f) d f}{\int_{(\text {band })} Y(f) d f}\right],
$$

where $Y(f)$ is the power spectral density of the received signal. However, in this work we use the ACLR (Adjacent Channel Leakage Ratio) which represents a similar concept but it is more appropriate, as it is a magnitude which is in the LTE-A especifications [6].

The transmitted BBU LTE signal has originally a ACLR of $51.6 \mathrm{~dB}$ and an RMS EVM of $0.1 \%$. As far as the efficiency is considered, the higher the number of levels in the partition, the better the total efficiency. This stems from the fact that every sample is transmitted using the right degree taking into account the efficiency. Therefore, the computational efficiency increases while the linearization efficiency is maintained. Obviously, the computed DPDs are not the best regarding
ACLR performance, but the price to pay in order to reduce complexity is low (1.6 dB in terms of ACLR) and $0.2 \%$ in EVM. Finally, Fig. 5 shows the nonlinear AM curve, the corrected and without DPD power spectral densities and the EVM corresponding to a subcarrier in the LTE signal with and without correction. They confirms that the DPD, despite the reduction in terms of complexity properly works.

\section{CONCLUSION}

A complexity reduction technique based on a variable degree polynomial predistorter is proposed in this paper. The model parameters are optimized by means of an Integer Genetic Algorithm, which computes the degrees to maximize the tradeoff between computational complexity and performance. The obtained results show that it is possible to achieve a reduction of operations with a penalty of $1.6 \mathrm{~dB}$ in ACLR and negligible impact on the EVM.

\section{ACKNOWLEDGMENT}

This work was supported by the Spanish Government (Project TEC2014-58341-C4-2-R from MICINN and FEDER).

\section{REFERENCES}

[1] Thomas Pfeiffer, "Next Generation Mobile Fronthaul and Midhaul Architectures". Journal of Optical Communications and Networking Vol. 7, Issue 11, pp. B38-B45 (2015).

[2] N. Alic, "Cancellation of nonlinear impairments in fiber optic transmission systems," OFC 2016, paper Tu2E.1 (2016).

[3] Kim, Jiwoo, P.Roblin,D. Chaillot,"A Generalized Architecture for the Frequency- Selective Digital Predistortion Linearization Technique",IEEE Trans. Microw. Theory Tech. Vol. 71, Issue 1, pp. 596-605, Jan 2013

[4] A. S. Tehrani, S. Afsardoost, T. Eriksson, M. Isaksson, and C. Fager, "A comparative analysis of the complexity/accuracy tradeoff in power amplifier behavioral models," IEEE Trans. Microw. Theory Tech., vol. 58, no. 6, pp. 1510?1520, May 2010.

[5] Guan, Lei and Zhu, Anding, "Optimized low-complexity implementation of least squares based model extraction for digital predistortion of RF power amplifiers," IEEE Trans. Microw. Theory Tech., vol. 60, no. 3, pp. 594?603, May 2010.

[6] 3GPP TSGRAN "User Equipment (UE) Radio Transmission and reception (FDD)”, Technical Specification ETSI TS136.101 V9.4.0, 2010. 